\title{
GOATS'2002
}

\section{Multi-AUV Cooperative Behavior for Littoral MCM and REA Concurrent Mapping and Localization}

\author{
Henrik Schmidt \\ John Leonard \\ Department of Ocean Engineering \\ Massachusetts Institute of Technology \\ Cambridge, MA 02139 \\ phone: (617) 253-5727 fax:(617) 253-2350 email: henrik@ keel.mit.edu \\ Grant Number: N000149710202 \\ http://acoustics.mit.edu/arctic0/henrik/www/home.html
}

\section{LONG-TERM GOAL}

Develop environmentally adaptive sonar concepts for autonomous underwater vehicle networks for detection and classification of proud and buried targets in very shallow water.

\section{OBJECTIVES}

The objective of this sub-project is to develop the fundamental technology for operating multiple autonomous underwater vehicles in a coordinated, cooperative manner within the AOSN framework, with application to mine countermeasures and rapid environmental assesment in shallow and very shallow water. Specifically, this component of GOATS explores the potential of Concurrent Mapping and Localization (CML) using onboard sonar systems to navigate a fleet of cooperating AUVs in unmapped environments.

\section{APPROACH}

The development of GOATS (Generic Ocean Array Technology Sonar) is a highly interdisciplinary effort, involving experiments, and theory and model development in advanced acoustics, signal processing, and robotics. The center piece of the research effort is the GOATS'2000 Joint Research Program (JRP) conducted by SACLANTCEN and MIT with ONR support, which was scheduled to finish in Aug. 2001, but which has been extended with 5 years, formally incorporated in the SACLANTCEN Program of Work. Building on the experience of the highly succesful GOATS'98 pilot experiment [2] and the GOATS'2000 experiment, the JRP continues with a series of experiments, with the two major ones being planned for 2002 and 2004, and modeling and simulation work to explore the potential of autonomous underwater vehicle networks as platforms for new sonar concepts exploring the full 3-D acoustic environment of VSW. The modeling effort is centered around the OASES environmental acoustic modeling framework developed at MIT [1,4]. OASES is a widely distributed suite of models covering a variety of ocean waveguide and source/receiver representations. Thus, the most recent developments are computational modules for full wave theory modeling of mono-and bistatic reverberation in shallow water waveguides. In collaboration with SACLANTCEN the waveguide reverberation code OASSP has been modify to consistently model the mono- and bistatic 


\section{Report Documentation Page}

Form Approved

OMB No. 0704-0188

Public reporting burden for the collection of information is estimated to average 1 hour per response, including the time for reviewing instructions, searching existing data sources, gathering and maintaining the data needed, and completing and reviewing the collection of information. Send comments regarding this burden estimate or any other aspect of this collection of information,

including suggestions for reducing this burden, to Washington Headquarters Services, Directorate for Information Operations and Reports, 1215 Jefferson Davis Highway, Suite 1204, Arlington

VA 22202-4302. Respondents should be aware that notwithstanding any other provision of law, no person shall be subject to a penalty for failing to comply with a collection of information if it

does not display a currently valid OMB control number.

1. REPORT DATE

30 SEP 2002

2. REPORT TYPE

3. DATES COVERED

00-00-2002 to 00-00-2002

4. TITLE AND SUBTITLE

GOATS'2002, Multi-AUV Cooperative Behavior for Littoral MCM and REA Concurrent Mapping and Localization

6. AUTHOR(S)

5a. CONTRACT NUMBER

5b. GRANT NUMBER

5c. PROGRAM ELEMENT NUMBER

5d. PROJECT NUMBER

5e. TASK NUMBER

5f. WORK UNIT NUMBER

7. PERFORMING ORGANIZATION NAME(S) AND ADDRESS(ES)

Department of Ocean Engineering,,Massachusetts Institute of 8. PERFORMING ORGANIZATION REPORT NUMBER

Technology,,Cambridge,MA, 02139

9. SPONSORING/MONITORING AGENCY NAME(S) AND ADDRESS(ES)

10. SPONSOR/MONITOR'S ACRONYM(S)

11. SPONSOR/MONITOR'S REPORT

NUMBER(S)

12. DISTRIBUTION/AVAILABILITY STATEMENT

Approved for public release; distribution unlimited

13. SUPPLEMENTARY NOTES

14. ABSTRACT

15. SUBJECT TERMS

16. SECURITY CLASSIFICATION OF:

a. REPORT

unclassified b. ABSTRACT

unclassified c. THIS PAGE

unclassified
17. LIMITATION OF ABSTRACT

Same as

Report (SAR)
18. NUMBER 19a. NAME OF

OF PAGES

15 RESPONSIBLE PERSON

Standard Form 298 (Rev. 8-98) Prescribed by ANSI Std Z39-18 
reverberation from interface roughness and seabed volume inhomogeneities in azimuthally symmetric sonar scenarios [6]. Another module, OASES-3D provides wave-theory modeling of the full 3-D acoustic environment associated with mono- and bi-static configurations in VSW with aspectdependent targets and reverberation features [3,4]. OASES-3D incorporates environmental acoustic features specifically associated with bi-static sonar concepts in shallow water, including aspectdependent target models, seabed porosity, and scattering from anisotropic seabed roughness such as sand ripples. The validation of these models is one of the major objectives of the GOATS JRP with SACLANTCEN.

In addition to the acoustic research, the continuation of GOATS involves a significant effort addressing the fundamental robotics issues associated with the collaborative operation of multiple autonomous underwater vehicles in shallow water, including navigation, inmter-platform acoustic communication, and adaptive, cooperative behavior. Specifically the current effort explores the development of Concurrent Mapping and Localization (CML) algorithms for networks of AQUV-s, and the implementation of efficient inter-vehcle acoustic communication protocols enabling the cooperative behavior which is crucial to the implementation of the GOATS concept.

\section{WORK COMPLETED}

\section{GOATS’2002 Experiment}

The main component of the FY02 effort was the planning and execution of the BP-02/MASAI-02 experiments, carried out jointly with SACLANTCEN under the International Cooperation on AUV Technology Joint Research Program. MIT participated in the BP-02 (Battlefield Preparation) Rapid Environmental Assessment (REA) experiment, May 25-June 2, 2002, and the MASAI-02 Multi-AUV Cooperative and Adaptive Detection and Classification of Buried Targets experiment, June 4-June 18, 2002.

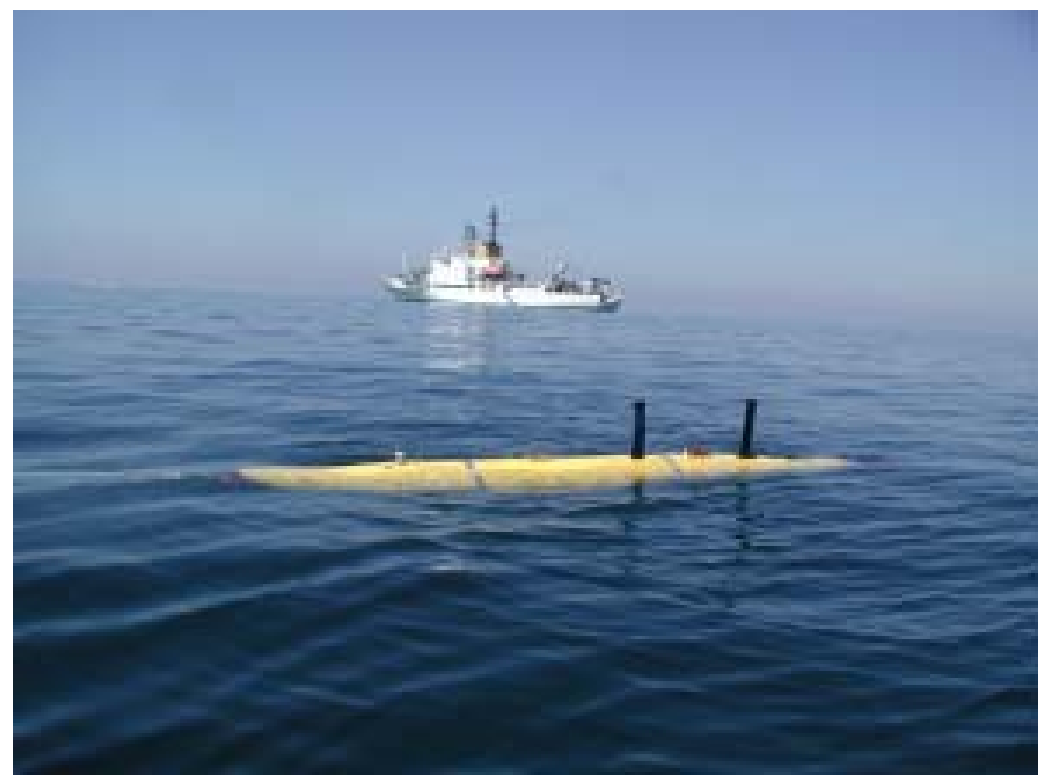

Figure 1: MASAI-02: Caribou Odysssey III AUV ready for launch, with $R / V$ Alliance in background 


\section{Objectives}

The objective of the MIT component of BP-02/MASAI-02 was to develop the fundamental technology enabling the use of multiple, cooperating AUV's for autonomous, concurrent detection and classification of proud and buried targets using low-frequency, bottom-penetrating synthetic aperture sonar technology. The main objective of this research is the development SAS processing of sonar signals recorded concurrently by mono- and bi-static apertures, and the associated auto-focusing. Other enabling technology that were be tested and demonstrated are new navigation and communication technology for multiple Autonomous Underwater Vehicles performing cooperative surveys. Specifically the MIT components of BP-02/MASAI-02 attempted to demonstrate the field performance of new Concurrent Mapping and Localization (CML) algorithms currently being developed at MIT. The acoustic communication effort demonstrated inter-vehicle acoustic communication for coordinated seabed surveys using a mono-static synthetic aperture sonar on one ASUV and a video camera on the other. The final objective was to demonstrate autonomous, adaptive vehicle behavior based on real-time onboard processing of sonar data, with one or both vehicles being adaptively redirected based on on-board, conventional and SAS processing. Even though all of these objectives were not met completely, significant progress was made in all areas.

\section{Equipment}

The original plan was to deploy three AUV's in BP-02/MASAI-02, with up to two being deployed concurrently for dual-vehicle mission tests, but the second of two new Bluefin Odyssey-III AUVs was not completed in time due to delay in funding. As a result, MIT deployed only two AUVs. The main AUV platform was be a new, modular Odyssey III, Caribou, recently acquired from Bluefin Robotics by MIT. It was operated with two different payload sections, one being an Edgetech combined SSSSBP sonar, and the other being a SAS sonar system consisting of interchangeable nose-mounted 2x8element and 1x16-element nose arrays built at SACLANTCEN, and center payload section containing a dedicated data acquisition system and an Edgetech $4-16 \mathrm{kHz}$ SSB profiler source used as active element. The source is re-configurable to provide 2-sided, insonification grazing angles in the range 20-90 degrees.

As a second vehicle MIT operated a refurbished Odyssey II, Xanthos. This vehicle was equipped with a video camera. The two vehicles operated the same new vehicle control software, MOOS, currently being developed at MIT. They also operated the same navigation and communication hardware and were therefore interchangeable in regard to all the navigation and communication work planned for MASAI-02. 

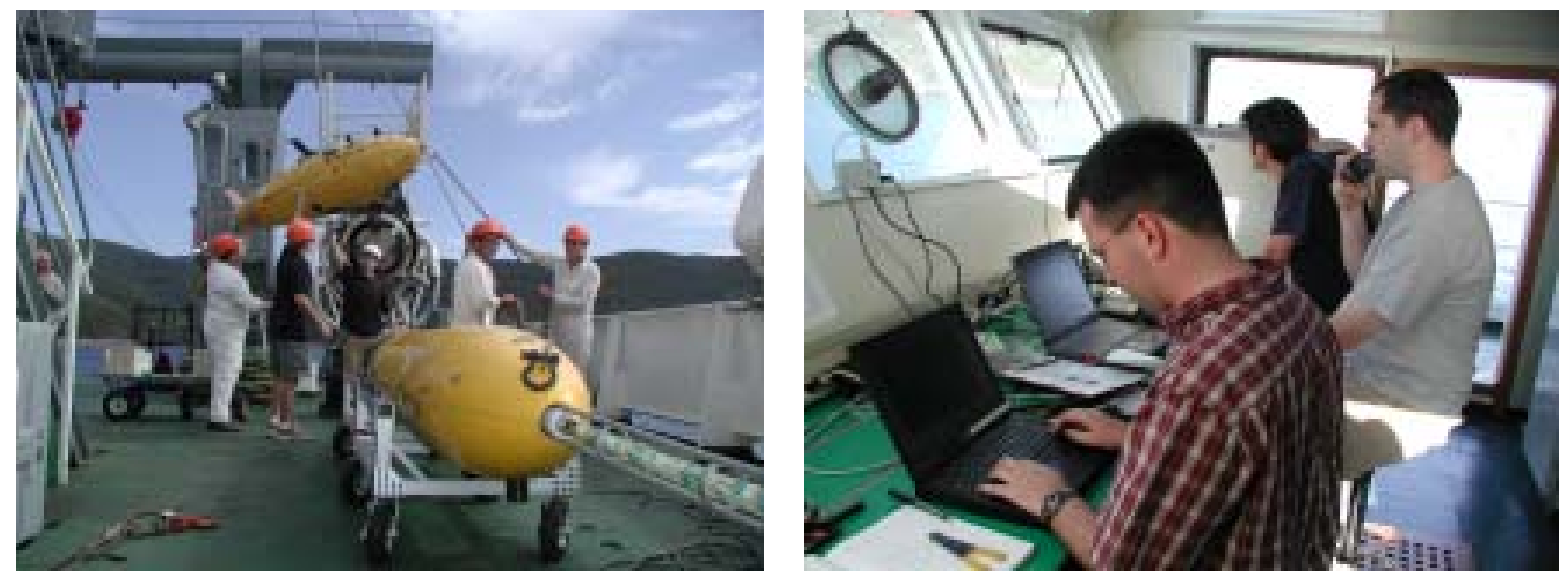

\section{Operation}

The AUVs were operated by a team of engineers from Bluefin Robotics and the MIT Sea Grant AUV Laboratory. The main difference in operation procedure from earlier GOATS experiments was the use of the aft bridge of Alliance as operations center for all MIT AUV operations. This was made possible by the new MOOS operating system allowing full remote radio programming and control of the AUVs. In earlier experiments all operations were conducted from MSL, without visual contact with the vehicles, not even on the surface. The use of the aft bridge provided a much more efficient operational environment, and towards the end of the experiment missions could be turned around in very short time, and a total of more than 500 dives were completed by the MIT team during BP-02 and MASAI02, most of them short, but for the type of AUV research performed by MIT, focusing on the developing the fundamental technology, many short missions in rapid succession is crucial.

The early part of the cruise was hampered by rough seas and windy conditions in Framura, and only one half day of full operations was achieved during the first week of MASAI-02, June 4.

SACLANTCEN was requested to obtain clearance for Biodola Bay, Elba. The clearance was given for June 7 and onwards. Two and a half days of operations were completed in Biodola before conditions improved, and Alliance returned to Framura to resume scheduled operation June 10. The clearance for Biodola required all equipment and Alliance to be out of the bay by 1900 each day, which affected operations somewhat. Specifically, the acoustic navigation and communication network had to be redeployed every morning, and re-calibrated. However, the decision to go to Elba was the right one from MIT's point of view. Significant progress was made on both navigation and acoustic communication, as well as on dynamic control of Caribou with the 1x16-element nose array. During the last week in Framura, June 10-17, conditions were excellent and most of the objectives were met. During this entire period, the new BIB GPS tracking system was deployed as well, with the buoys acting as floats for the LBL navigation network.

\section{ACCOMPLISHMENTS}

\section{MOOS - Mission Oriented Operation Suite}

One of the main MIT objectives was the development of the fundamental control algorithms that allows fleets of AUVs to cooperate on adaptive sampling in the ocean environment. A key component of such algorithms is the capability of reacting to socalled $3^{\text {rd }}$ party requests where a process external 
to the vehicle control, e.g. one running a sensor system or one running another AUV can request changes in behavior. These requests can arrive via the internal AUV Ethernet, or via acoustic modems. Native AUV software, including the one developed by Bluefin Robotics for the Odyssey III vehicle are in general developed for easy setup of survey patterns, e.g for side-scan imaging of a large area of seabed, and does not directly support $3^{\text {rd }}$ party requests. Also, for safety and warranty reasons the commercial control software is in general based on a tightly closed architecture, the custumization of which is time consuming and expensive, requiring tight cooperation with the manufacturer. As a result, MIT decided in the fall of 2002 to develop a new, open AUV software architecture, MOOS - Mission Oriented Operating Suite with the following characteristics

- An alternative, small and fast source code solution for AUV operation

- $\quad$ Fully distributed, platform independent

- Mission Simulation, Playback and Reprocessing

- $\quad$ On the fly mission task arbitration and scheduling

- $\quad 3$ rd party task invocation - for example

○ via acoustic modem - mission start/stop - redirect

○ From scientific payload - "orbit this area" (if you can)

○ From another vehicle - "my survey finished - come take photos"

- Remote control and "at sea" mission specification

- Start MOOS once - processes just "refresh" upon user changes. Very stable.

Since the first line of code was written by the developer, Dr. Paul Newman of MIT, in September 2001, MOOS has been implanted and tested on several different autonomous platforms, including an autonomous surface craft, and Odyssey II AUV, and two different land-robots. After delivery of the new Odyssey III, Caribou, to MIT in Dec. 2001, MOOS was integrated and underwent preliminary tests in late March, 2002 shortly before shipment to the Centre of all MIT equipment, and as a result, MOOS had only about 20 minutes of operation time on Caribou at the beginning of BP-02/MASAI-02. A significant part of the operation time during the experiment was therefore used on advancing the capabilities of MOOS to perform adaptive cooperative behaviors, including the following specific at-sea developments

- Vehicle Control

- Dynamic control of vehicle with SAS payload--interesting hydrodynamic properties

○ Execution of Survey's, Zamboni's, Tracklines, Waypoints

○ Pilot invoked "come home" facility and on surface remote control

- Navigation

○ Closed loop LBL/DVL/Compass navigation

○ Closed loop LBL net sharing for two AUV's using Xanthos and Caribou 
- Acoustic Modem

○ Remote control of vehicle - mission start stop redirect

○ Active, unsolicited remote query of vehicle status - "tell me battery state" etc

○ Broadcast of state - eg "current position is..." or "current task is....." etc

- $\quad$ Payloads

- Integration and control of low-frequency SAS payload from other MOOS processes

○ Integration and control of Edgetech SSS/SBP payload.

- Missions

- Over 500 complete mission executed. Many short (<2 min) but last days average mission time $\sim 30$ minutes.

- Each mission was planned, executed and logged without a MOOS restart.

○ Very fast turn around: an ideal research platform

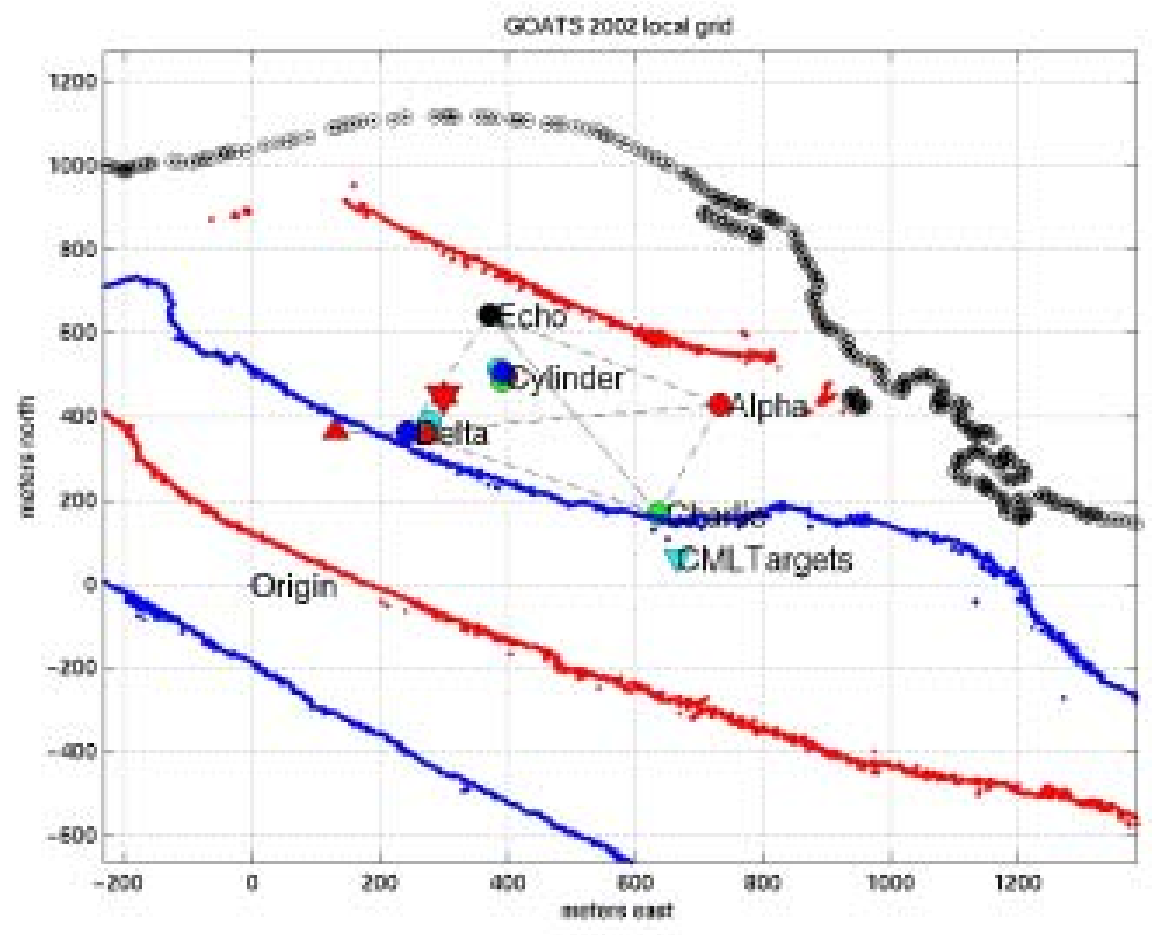

Figure 2. Framura test area with MIT LBL navigation and GIB tracking network. 


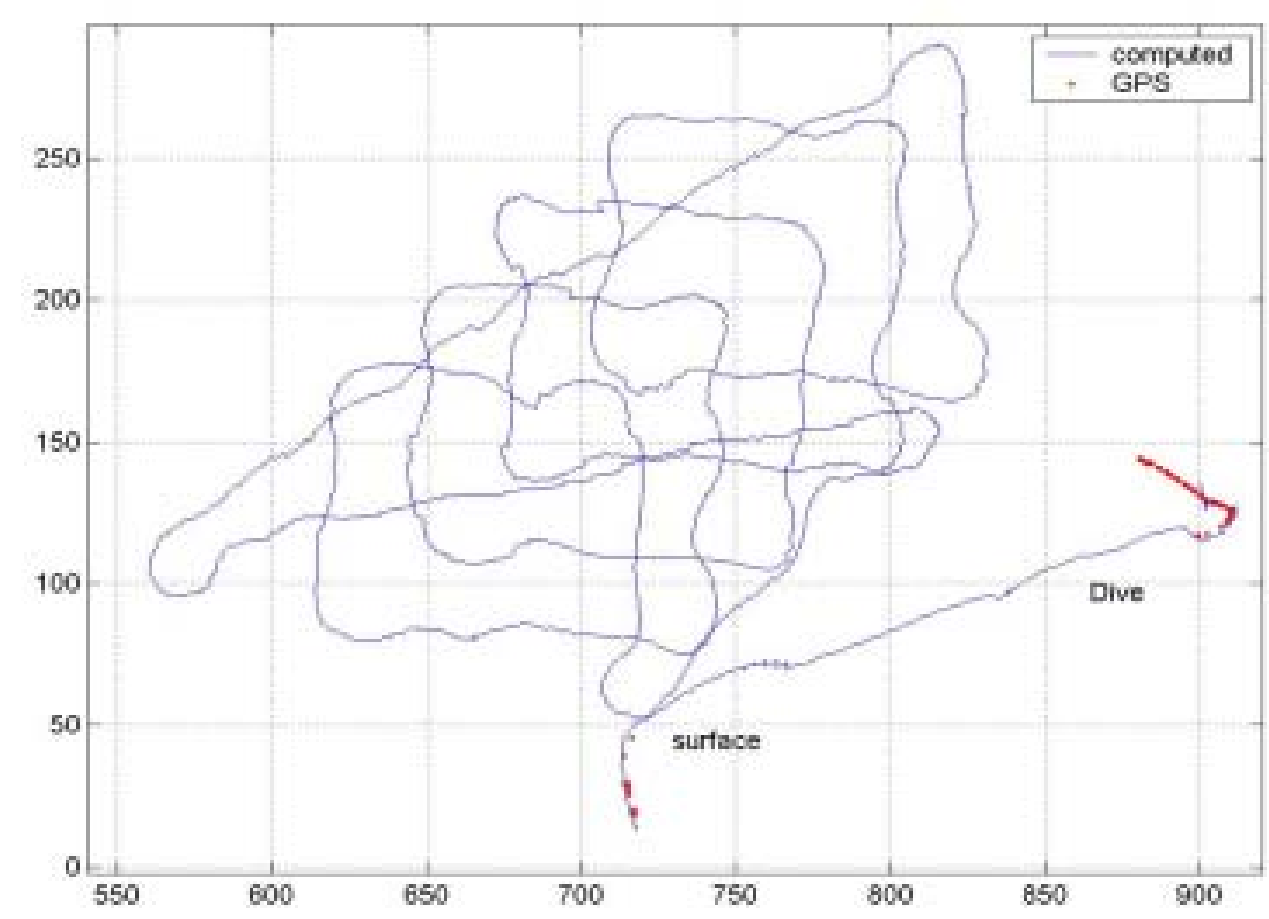

Figure 3: AUV navigation during SAS survey. Blue track indicates the onboard navigation result while the red dots indicate the GPS fixes while at the surface before the dive and after the surfacing, confirming navigation accuracy better than 1 meter throughout the mission.

\section{Navigation}

One of the core components of MOOS is the navigation algorithm, fusing several independent navigation sensors into an optimal vehicle navigation involving multiple cooperating platforms. Caribou used an on-board DVL, compass, and GPS (while surfaced), together with a Sonardyne longbaseline acoustic navigation network, deployed in the Framura test area as shown in Fig. 2.

Fig. 3 shows a trajectory with a so called zamboni search pattern by Caribou in an area where many concrete blocks were observed in previous surveys as shown in Fig. 1 . The vehicle was using the SBP source with the $2 \times 8$ nose array during the survey. The blue track indicates the vehicle navigation using all available sensors, fused by an extended Kalman filter, while the red dots indicate GPS fixes on the surface. The mission started at the easternmost point and ended at the southernmost point. As is obvious from the figure, the vehicle navigation achieved a position uncertainty at the end of the survey of less than one meter. This in spite of the fact that the LBL data were highly contaminated by false triggers generated by the low-frequency SAS system

\section{Low-frequency SAS Sonar}

Another objective of the MIT effort was to test a new low-frequency SAS sonarusing the two arrays built by the Centre and mounted in the nose of Caribou. A dedicated acoustic acquistion systm has been developed by MIT and integrated in the Odyssey III as shown in Fig. 4 

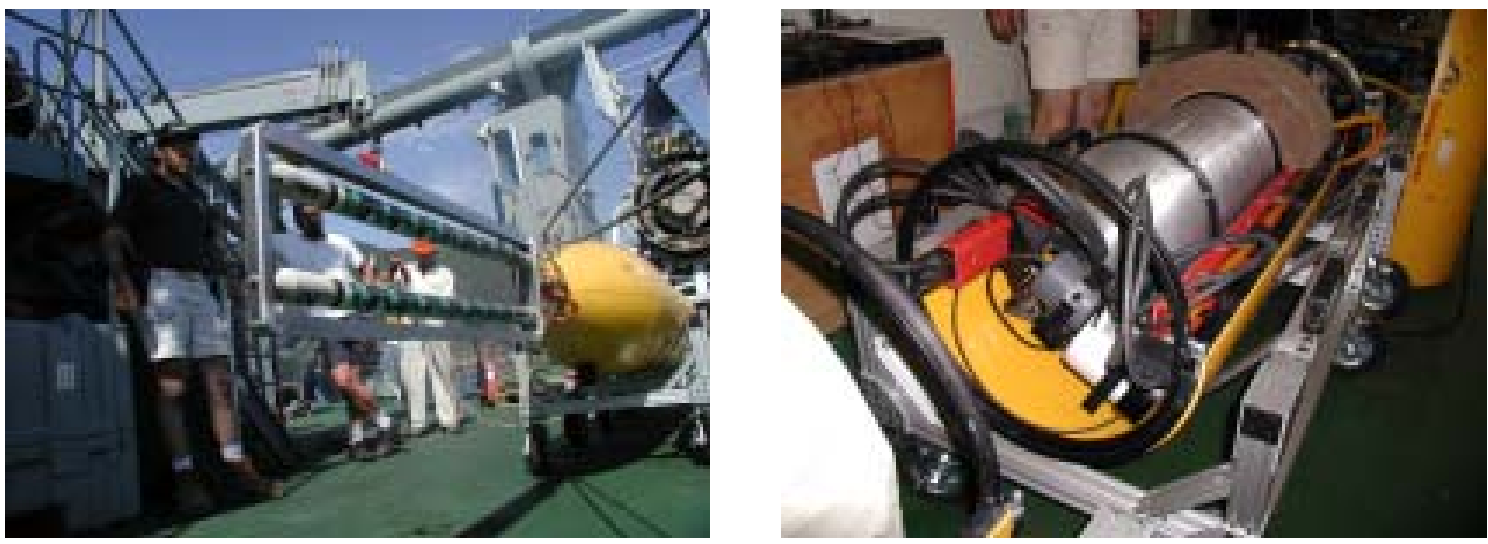

Figure 4. Low-frequency SAS sonar with nose-mounted $2 x 8$ element array and acquisition payload with 4-20 kHz SBP source and 16-chanmnel DSP acquisition system.

The new SAS system developed at MIT has the following characteristics

- $\quad 16$ Array Input Channels, $100 \mathrm{KHz}$ Sampling

- $\quad$ Simultaneous 16-Channel Sigma-Delta A/D Conversion

- $\quad$ Texas Instruments C6701 Floating-Point DSP

- Integrated $230 \mathrm{KHz}$ D/A Converter

- 200-Watt, 4-24 KHz Acoustic Source

- $\quad$ Adjustable 20-70 Degrees from Horizontal in 5 Degree Increments

- $\quad 233 \mathrm{MHz}$ Pentium Host CPU Board with Ethernet

- $\quad 30$ GB Ultra DMA/100 Hard Disk for Real-Time Data Storage

- $\quad$ Time Tags Using GPS-Synchronized Rubidium Oscillator

The SAS sonar was integrated into Caribou and the MOOS software architecture before and during the cruise,

- $\quad$ Fully Integrated Odyssey-III Payload Section

○ Power and Ethernet Connectivity to Main Vehicle

- $\quad$ Full Software Integration with MOOS on Main Vehicle

- Startup/Shutdown/Real-Time Status

- MOOS-consistent file names

○ Real-Time Control of Sonar Parameters

- Start/Stop Acquisition

- Source Ping Rate

- Sampling Period

○ Automatic Sharing of Real-Time Processed Data 
- Target Acquisitions/Tracking

- $\quad$ Adaptive Vehicle Control via Third-Party Requests

○ Real-Time Detection/Classification Algorithm on Host CPU

The new sonar system and its software integration was tested extensively during the cruise, with the results summarized as follows

- $\quad$ Collected Sonar Data on Eight Separate Days

○ Approximately $20 \mathrm{~GB}$ of Data from 70 Missions

- $\quad$ No Significant Hardware Failures

- $\quad$ Proved Real-Time Mine Detection From an AUV is a Viable Concept

○ Successfully ran real-time, autonomous detection algorithm

\section{Concurrent Mapping and Localization}

Sonar data is not easy to interpret. Reliable, sonar based perception is crucial if AUVs are to perform high level tasks. Based on empirical evidence, many AI researchers advocate passing data through a series of specialized functions, each extracting/searching for a specific physically inspired characteristic or modality in the data. Edge detection and velocity estimation using Doppler shifts are typical examples.

Building on stochastic mapping with working memory, a form of Concurrent Mapping and Localization (CML) in development at MIT that allows delayed decision making and partially observable feature mapping, we have been developing a sequence of steps appropriate for sonar perception. At the various stages, beam forming, matched filtering, applying binary geometric constraints, applying dynamic constraints, applying target strength constraints, and Mahalanobis testing are performed.

\section{RESULTS}

CML has been successfully demonstrated using sonar in the MIT testing tank. In the example shown in Fig. 5, a robot performed CML using a curved object at an unknown location. On the left is the Cartesian projection of raw sonar data. In the middle, the range components of those measurements are plotted as a function of time. On the left, the robot's measurement groupings are shown. These groupings were sufficient for CML. 

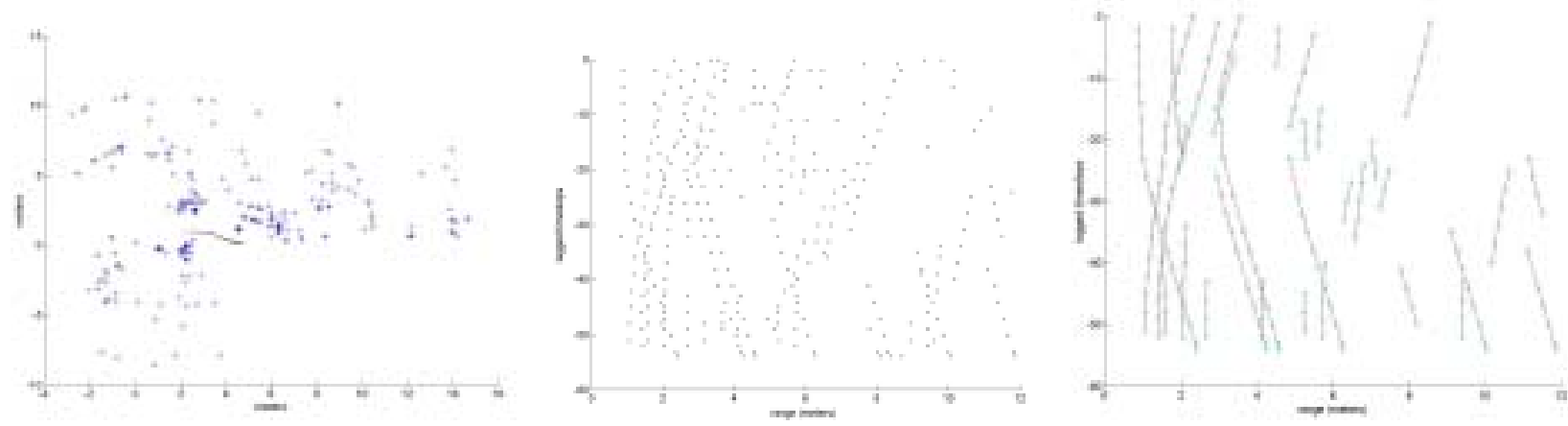

Figure 5. Tank demonstration of Concurrent Mapping and Localization

A similar approach is currently being applied to process the GOATS 2002/MASAI-02 data, examples of which are shown in Fig. 6. At present, the system is limited to roll and pitch stabilized vehicles, but the equations for target tracking from a six degree of freedom vehicle is being derived. With the high quality GOATS data shown below, it should be straightforward to extract measurement sets like those shown above.
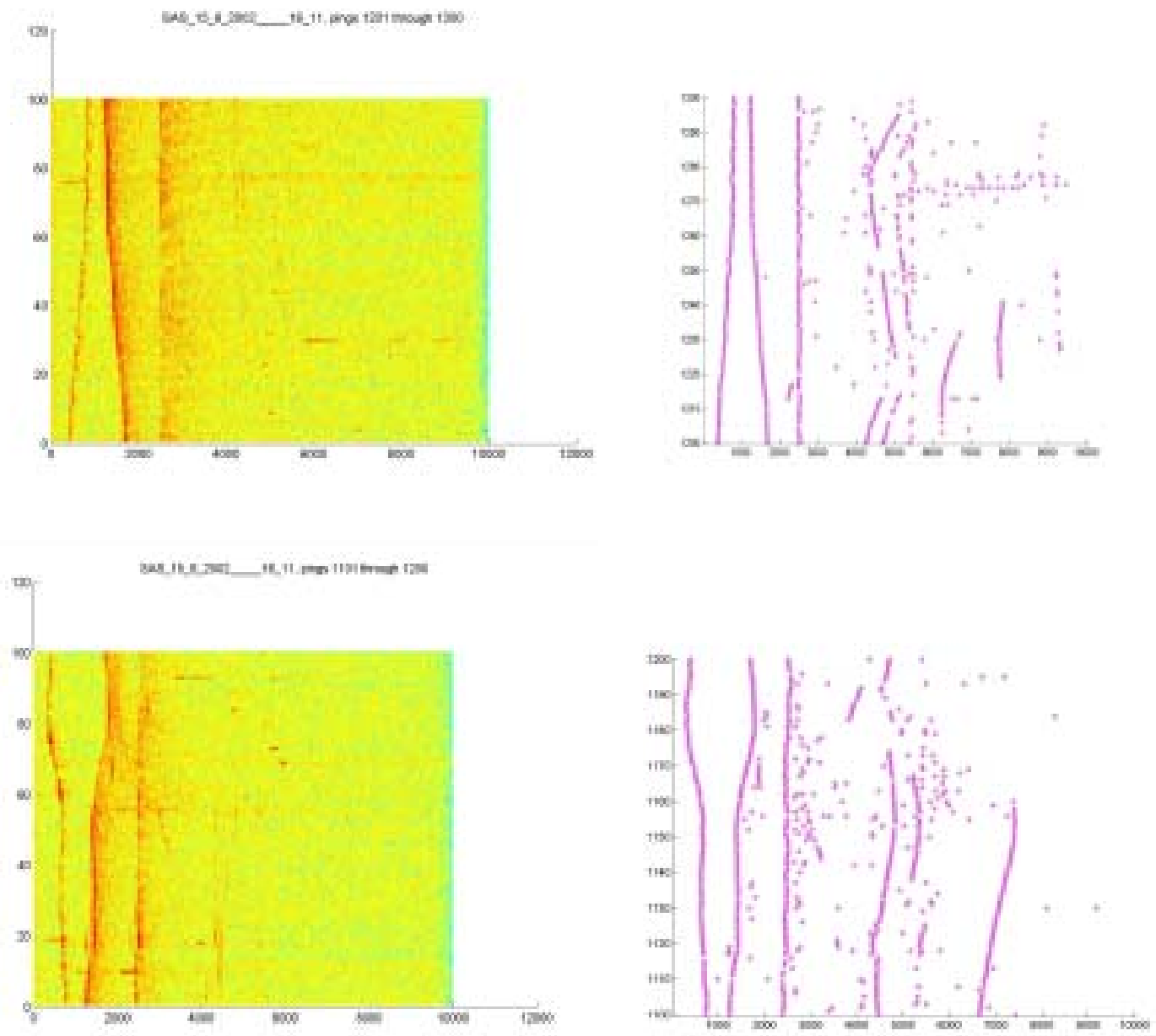

Figure 6. Examples os SAS data collected during MASAI-02, with associated detections. The first three arrivals in both datasets are the surface bounce, the bottom bounce, and the bathymetry (surface-bottom) arrival. The later arrivals constitute targets detected by the beamformingdetection-tracking algorithm developed by the MIT GOATS group. 


\section{IMPACT/APPLICATION}

The long-term impact of this effort is the development of new sonar concepts for VSW MCM, which take optimum advantage of the mobility, autonomy and adaptiveness of the AOSN. For example, biand multi-static, low-frequency sonar configurations are being explored for buried mines in VSW, with the traditional high-resolution acoustic imaging being replaced by a 3-D acoustic field characterization as a combined detection and classification paradigm, exploring spatial and temporal characteristics which uniquely define the target and the reverberation environment.

\section{TRANSITIONS}

The GOATS AUV effort has been and is conducted in cooperation with the MIT Sea Grant AUV Laboratory and Bluefin Robotics, a spin-off from the MIT Laboratory. Bluefin is currently developing and building the Odyssey III Battlefield Preparation AUV for ONR, and similar MCM platforms for the Coastal Systems Station (CSS) and QinetiQ (UK), building in part on the experience and results from the GOATS effort.

The results of th multi-vehicle navigation, communication and cooperative behavior is being transitioned into the Autonomous Operations Future Naval Capabilities (AOFNC) project Demonstratio of Undersea, Autonomous Operation Capabilities and related Technology Development. John Leonard is the MIT PI of this joint project with Bluefin Robotics and the Naval Undersea Warfare Center..

The OASES acoustic propagation framework continues to be maintained and expanded. It is continuously being exported or downloaded from the OASES web site (http://acoustics.mit.edu/arctic0/henrik/www/oases.html), and used extensively by the community as a reference model for ocean seismo acoustics in general.

\section{RELATED PROJECTS}

This effort is part of the US component of the GOATS 2000 Joint Research Project (JRP) with the SACLANT Undersea Research Centre. The MIT GOATS effort is funded jointly by ONR codes 3210A (Simmen), 3210E (Swean), 321TS (Johnson), and 322OM (Curtin).

The GOATS effort is strongly related to the ONR Autonomous Ocean Sampling Network (AOSN) initiative completed in FY00. Thus the GOATS'98 experimental effort was funded in part by the AOSN MURI, (PI: J. Bellingham). In terms of the fundamental seabed penetration physics there are strong relations to the High-Frequency Bottom Penetration DRI (PI: E. Thorsos). This effort also builds on acoustic modeling efforts initiated under the Sea-Ice Mechanics Initiative (SIMI), and continued under funding from ONR code 321OA (Simmen).

With its heavy focus on Synthetic Aperture Processing approaches and their extension to bi- and multistatic configurations in multipath SW VSW environments, there are strong relations to the ONR SASSAFRASS project (code 321TS and 3210A). 
The OASES modeling framework being maintained and upgraded under this contract is being used intensively as part of the MIT AREA (Adaptive Rapid Environmental Assesment) component of the new ONR "Capturing Uncertainty” DRI (Grant \# N0014-01-1-0817), aimed at mitigating the effect of sonar performance uncertainty associated with environmental uncertainty by adaptively deploying environmental assesment resources.

\section{REFERENCES}

1. Schmidt, H. “OASES V 2.7 User's Guide”, Massachusetts Institute of Technology, 2000.

2. Schmidt, H., Maguer, A, Bovio, E., Fox, W.L.J., LePage, K., Pace, N.G., Hollett,R., Guerrini, P., Sletner, P.A., Michelozzi, E., Moran, B. and Grieve, R. "GOATS'98 - AUV Network Sonar Concepts for Shallow Water Mine Countermeasures," SACLANT Undersea Research Centre, Report SR-302, 1998.

3. Schmidt, H. and Lee, J.Y, "Physics of 3-D scattering from rippled seabeds and buried targets in shallow water," Journal of the Acoustical Society of America, 105(3), 1605-1617, 1999.

4. Schmidt, H. Three-dimensional scattering from buried targets in shallow water. In: Bovio, E., Schmidt, H., editors. GOATS 2000, SACLANTCEN CP-46, 2002Tesei, A., Lim, R., Maguer, A., Fox, W.L.J., Schmidt, H. Measurements of acoustic scattering from partially and completely buried spherical shells. Journal of the Acoustical Society of America Submitted, 2001.

\section{PUBLICATIONS}

J. R. Edwards, H. Schmidt and K. LePage, Bistatic synthetic aperture target detection and imaging with an AUV, IEEE Journal of Oceanic Engineering 26(4): 690-699, 2001.

LePage, K., Schmidt, H. Bistatic synthetic aperture imaging of proud and buried targets using an AUV, IEEE Journal of Oceanic Engineering (In press), 2002.

LePage, K., Schmidt, H. Spectral integral representations of monostatic backscattering from threedimensional distributions of sediment volume inhomogeneities. Journal of the Acoustical Society of America, (In Press), 2002.

Tesei, A., Lim, R., Maguer, A., Fox, W.L.J., Schmidt, H. Measurements of acoustic scattering from partially and completely buried spherical shells. Journal of the Acoustical Society of America (In press), 2002.

Schmidt, H. Three-dimensional scattering from buried targets in shallow water. In: Bovio, E., Schmidt, H., editors. GOATS 2000, SACLANTCEN CP-46, 2002.

H. Schmidt and J.R. Edwards, GOATS: Multiplatform Sonar Concept for Coatsal Mine Countermeasures. In: A.C. Schultz and L.E. Parker editors: Multi-Robot Systems: From Swarms to Intelligent Automata. Kluwer Academic Publishers, 2002. 
J. J. Leonard, R. J. Rikoski, P. M. Newman, and M. Bosse. Mapping Partially Observable Features from Multiple Uncertain Vantage Points. To appear in International Journal of Robotics Research.

J. D. Tardos, J. Neira, P. M. Newman, and J. J. Leonard. Robust Mapping and Localization in Indoor Environments using Sonar Data. To appear in International Journal of Robotics Research.

M. Bosse, S. Teller, R. Rikoski, and J. Leonard. Omni-directional Structure from Motion Using Vanishing Points and 3D Lines. To Appear in Visual Computer.

Richard J. Rikoski, John J. Leonard, and Paul M. Newman. Stochastic Mapping Frameworks. Proceedings of the 2002 IEEE International Conference on Robotics and Automation, May, 2002.

John W. Fenwick, Paul M. Newman, and John J. Leonard. Cooperative Concurrent Mapping and Localization. Proceedings of the 2002 IEEE International Conference on Robotics and Automation, May, 2002.

Paul M. Newman, John J. Leonard, J. Neira and J. Tardos. Explore and Return: Experimental Validation of Real Time Concurrent Mapping and Localization. Proceedings of the 2002 IEEE International Conference on Robotics and Automation, May, 2002.

W. Xu, TC Liu, and H. Schmidt, "Beamforming based on spatial-wavelet decomposition," Proc. of the IEEE Sensor Array and Multichannel Signal Processing (SAM) Workshop, August, 2002.

T.C. Liu and H. Schmidt. "Auv-based subbottom target detection and tracking." OCEANS 2002: Reflections of the past, visions of the future, Biloxi, MS, 2002. MTS/IEEE.

T.C. Liu and H. Schmidt. "Concurrent detection and localization of buried targets using track-beforedetect processing." 144th Meeting of the Acoustical Society of America, Cancun, Mexico, 2002. AIP.

J.R. Edwards and H. Schmidt. "Real-time classification of buried targets with teams of unmanned vehicles." OCEANS 2002: Reflections of the past, visions of the future, Biloxi, MS, 2002. MTS/IEEE.

M. W. M. G. Dissanayake, P. M. Newman, H. F. Durrant-Whyte, S. Clark, and M. Csorba. A solution to the simultaneous localization and map building (SLAM) problem, IEEE Transactions on Robotic and Automation, Vol 17, No 3, Pages 229-241, 2001

P. M. Newman and H. F. Durrant-Whyte. An Efficient Solution to the SLAM Problem Using Geometric Projections, Proceedings of the November 2001 SPIE conference, Boston, USA.

S. B. Williams, P. M. Newman, J. Rosenblatt, G. Dissanayake, and H. F. Durrant-Whyte. "Autonomous Underwater Navigation and Control". Robotica, vol 19, no 5, September, 2001 Pages 481-496.

J. J. Leonard, P. M. Newman, R. J. Rikoski, J. Neira, and J. D. Tardós. Towards Robust Data Association and Feature Modeling for Concurrent Mapping and Localization. Proceedings of the Tenth International Symposium on Robotics Research, Lorne, Victoria, Australia, November, 2001. 
M. Stojanovic, L. Freitag, J. Leonard, P. Newman. A network protocol for Multiple AUV

Localization. To appear in proceedings MTS/IEEE Oceans 2002 Conference, Biloxi, USA. 